\title{
A Statistical investigation of the invariant latitude dependence of unstable magnetospheric ion populations in relation to high $\mathbf{m}$ ULF wave generation
}

\author{
M. E. Wilson ${ }^{1}$, T. K. Yeoman ${ }^{1}$, L. J. Baddeley ${ }^{1}$, and B. J. Kellet ${ }^{2}$ \\ ${ }^{1}$ Department of Physics and Astronomy, University of Leicester, University Road, Leicester, LE1 7RH, UK \\ ${ }^{2}$ Rutherford Appleton Laboratory, Didcot, UK
}

Received: 7 December 2005 - Revised: 21 July 2006 - Accepted: 6 September 2006 - Published: 22 November 2006

\begin{abstract}
A statistical study is presented of the unstable proton populations, which contain the free energy required to drive small-scale poloidal mode ULF waves in the magnetosphere, observed at invariant latitudes of $60^{\circ}$ to $80^{\circ}$. The data are all in the form of Ion Distribution Functions (IDFs) amassed over $\sim 6$ years using the CAMMICE (MICS) instrument on the Polar spacecraft, and cover proton energies of $1 \mathrm{keV}$ to $328 \mathrm{keV}$. The free energy contained in the unstable, positive gradient regions of the IDFs is available to drive resonant wave growth. The results show that positive gradient regions in IDFs on magnetic field lines corresponding to the lower invariant latitudes in the range under study occur predominantly in the afternoon sector at proton energies of $5 \mathrm{keV}$ to $20 \mathrm{keV}$. In the morning and dawn sectors positive gradient regions are seen with a typical proton energy range of $5 \mathrm{keV}$ to $45 \mathrm{keV}$. While the proton energy peaks in the afternoon sector at around $\sim 7 \mathrm{keV}$ the morning sector has two peaks occurring at $\sim 10 \mathrm{keV}$ and $\sim 20-30 \mathrm{keV}$. The technique of Baddeley et al. (2004), employed to quantify the free energy in each IDF, found that as invariant latitude increased the free energy contained in the positive gradient regions fell. Positive gradient regions in the afternoon sector decrease in number with invariant latitude at a faster rate than those in the morning sector. The majority of positive gradient regions had free energy values of $>10^{10} \mathrm{~J}$ with many at the lowest invariant latitudes having free energies of in excess of $10^{11} \mathrm{~J}$. Positive gradient regions at proton energies of $>100 \mathrm{keV}$ are rarely observed, and have free energies of typically $<10^{9} \mathrm{~J}$, which is too small to produce high $m$ ULF waves of significant amplitude.
\end{abstract}

Keywords. Magnetospheric physics (Energetic particles, trapped; MHD waves and instabilities)

Correspondence to: M. E. Wilson

(mew6@ion.le.ac.uk)

\section{Introduction}

Ultra Low Frequency (ULF) waves, with frequencies in the $\mathrm{mHz}$ range, have two major generation mechanisms; those external and internal to the Earth's magnetosphere. Externally generated waves are characterised by having small magnitude effective azimuthal wave numbers, $m$ (i.e. they have a large scale size in the azimuthal direction) and azimuthally polarised magnetic field oscillations. These are the result of mechanisms such as magnetopause KelvinHelmholtz instabilities, impulsive solar wind pressure increases or solar wind buffeting. Conversely, internally generated waves are characterised by their small azimuthal scale size and high $m$ values and have radially polarised magnetic field oscillations. It is now widely accepted that these ULF waves are generated by wave-particle interactions between unstable ion distribution functions in the ring current and standing wave modes along closed geomagnetic field lines.

The drift and bounce motions of energetic particles, which constitute part of the global ring current, may lead to small fluctuations in the electric and magnetic fields in the magnetosphere and ionosphere if there is excess energy available to the wave. It has been suggested (e.g. Southwood, 1976; Hughes et al., 1978) that there is free energy available to be fed into waves when westward drifting ions have ion distribution functions (IDFs) containing a positive gradient region, i.e.:

$\frac{d f}{d W}=\frac{\partial f}{\partial W}+\frac{d L}{d W} \cdot \frac{\partial f}{\partial L}>0$

where $f$ is the ion distribution function, $W$ is the ion energy and $L$ is the $L$-shell. This condition will be satisfied when there is a positive gradient in $\partial f / \partial \mathrm{W}$, or a negative gradient in $\partial f / \partial L$ for westward propagating $(m<0)$ waves (Southwood, 1976), or both. Here we are concerned with single-spacecraft data and only have measurements of $\partial f / \partial W$. These nonMaxwellian (also known as "bump-on-tail") IDFs are commonly created by natural processes of the magnetosphere

Published by Copernicus GmbH on behalf of the European Geosciences Union. 
such as particle injections associated with substorm activity. Subsequent to such an injection the ions are subject to gradient-curvature and $\boldsymbol{E} \times \boldsymbol{B}$ drifts and move westwards, arriving at a given magnetic local time at different universal times due to the energy dependence of magnetospheric drift paths. Even under steady state conditions particles follow energy-dependent drift paths which can lead to the formation of bump-on-tail distributions (Cowley and Ashour-Abdallah, 1976; Ozeke and Mann, 2001); they may, at some point on their path, match the local resonance condition (Southwood et al., 1969), given by

$\omega_{\text {wave }}-m_{\text {wave }} \omega_{\text {drift }}=N \omega_{\text {bounce }}$

where $\omega_{\text {wave }}, \omega_{\text {drift }}, \omega_{\text {bounce }}$ are the angular frequency of the wave, proton azimuthal drift and proton bounce respectively and $N$ is an integer representative of the harmonic mode of the wave. Particles satisfying this condition see a constant wave electric field and if their IDF has a bump-on-tail feature the free energy contained within it is fed to a resonant wave mode by a process of inverse Landau damping, causing wave growth. Southwood (1976) showed that the $N=0$, \pm 1 resonances should be the dominant modes in the magnetosphere; the $N=0$ drift resonance requires a wave to have an odd-mode standing wave structure, while the $N= \pm 1$ driftbounce resonances require an even-mode standing wave.

Wave-particle interactions have been implicated as a source mechanism for a number of different categories of ULF wave observations. These include ground based observations of dusk sector storm time Pc5s (e.g. Allan et al., 1982, 1983) showing compressional waves of $m \sim-7$ to 80, and morning sector Giant Pulsation ( $\mathrm{Pg}$ ) observations on ground magnetometer arrays (e.g. Chisham and Orr, 1991). More recently radar observations have revealed a spectrum of such waves occur very commonly, ranging from storm time-like high $m \mathrm{Pc} 4$ waves events in the afternoon and noon sectors (Yeoman and Wright, 2001), to Pg-like events in the morning sector (Baddeley et al., 2002). Spacecraft observations have also revealed significant particle-driven wave populations, for example; Anderson (1993) and Anderson et al. (1990) found compressional Pc5 waves in the dusk sector in AMPTE/IRM data; Woch et al. (1990) found similar wave populations at geostationary orbit and Engebretson et al. (1992) investigated a number of radially polarised ULF wave events in the frequency range $5-15 \mathrm{mHz}$ using data from AMPTE/CCE, GOES 5 and GOES 6.

Many different theories (e.g. Southwood et al., 1969; Hasegawa, 1969), have addressed the problem of how particle energy is passed to a resonant wave mode, describing the conditions required in the ambient plasma of the ring current in which the wave is embedded and the wave symmetry requirements for wave growth. Although a variety of theories exist, all have the common element that they identify that the main energy source of high $m$ (i.e. $|m|>10$ ) pulsations as being magnetospheric ion populations. At present it is unclear as to which theory is correct in relation to the generation of westward propagating, poloidal mode, high $m$ waves. Originally remarked upon at the 1997 IAGA (International Association of Geomagnetism and Aeronomy) meeting (Takahashi, 1998) it was suggested that a study of "the distribution function of energetic particles associated with wave events" would be of import and benefit. Baddeley et al. (2004) completed a statistical study of magnetospheric particle data gathered from the Polar satellite looking at the occurrence of "bump-on-tail" ion distributions in the magnetosphere at $L$-shell locations 6-9. Motivated by this, this paper presents an expansion of that work, employing similar techniques, but using a significantly larger data set and extending the range of $L$-shells under investigation.

Knowing the location of ion distributions with a "bumpon-tail" feature in the magnetosphere, along with their properties, will allow for limits to be placed upon the type of ULF waves that can be expected to be observed in any particular region by various ground based, and space borne, instruments. Of chief importance are the particle energies involved in the resonant wave interactions. For a given wave, of frequency, $\omega_{\text {wave }}$, and azimuthal wave number $m$, there are multiple solutions, of the resonance equation (Eq. 2), pertaining to different wave modes. Such solutions show that for higher particle energies a fundamental standing wave mode is more likely in comparison to the lower energies expected in conjunction with a second harmonic mode structure.

A multitude of instruments have been used to characterise the ULF waves that are thought to be generated by the process of inverse Landau damping and particle-wave resonance, primarily located between $L$-shells 6-9. Geostationary spacecraft are in this latitude range and have been employed in studies of high $m$ waves (e.g. Anderson, 1993; Takahashi et al., 1987). High (magnetic) latitude ground based radar systems also cover the $L$-shells, such as SABRE (the Sweden And British auroral Radar Experiment; Nielsen et al., 1983; e.g. Yeoman et al., 1992) and CUTLASS (Cooperative UK Twin Located Auroral Sounding System, Milan et al., 1997; e.g. Baddeley et al., 2002). Ground based instruments, at fixed latitudes in this range, for example the DOPE HF system located near Troms $\varnothing$ (Doppler Pulsation Experiment, Wright et al., 1997; e.g. Yeoman et al., 2000) and the Troms $\varnothing$ heater (e.g. Baddeley et al., 2002), which produces artificially-induced ionospheric irregularities to provide ionospheric targets for systems such as CUTLASS have made observations of ULF waves at many MLTs. On occasion, satellites in polar orbits (e.g. Polar) have been used in conjunction with such ground based instruments (e.g. Wright et al., 2001) to combine direct wave observations with in situ magnetospheric particle data.

This paper will take a set of magnetospheric ion data and use a statistical analysis to find the prevalence of "bump-ontail" ion distributions at invariant latitudes in the $60^{\circ}-80^{\circ}$ range over all MLT locations. By including data on ion energies within the positive gradient region of the IDF, along with the occurrence rates of these distributions at various 
locations, conclusions will be drawn as to the category of resonant wave-particle interactions which can be expected in different areas of the magnetosphere. As a result this statistical analysis will provide a reference point for ULF wave observations from ground magnetometers and all the Northern hemisphere SuperDARN radars, with their near global coverage at the latitudes under investigation. Also, in addition to the Troms $\varnothing$ heater experiments described by Baddeley et al. (2002), HAARP (High-frequency Active Auroral Research Programme, Rodriguez et al., 1998) and SPEAR (Space Plasma Exploration by Active Radar, Robinson et al., 2006), located at $63.1^{\circ}$ and $75.1^{\circ}$ magnetic latitude respectively, can be used to provide more sources of artificial backscatter to observe such ULF wave activity.

\section{Instrumentation}

The database created for the study is compiled from the omnidirectional IDFs recorded using the Magnetospheric Ion Composition Sensor (MICS) of the Charge And Mass Magnetospheric Ion Composition Experiment (CAMMICE) particle detector on board the Polar satellite (Wilken et al., 1992). Launched on 24 February 1996 the satellite was placed into a highly elliptical orbit with an apogee of 9 Earth radii $\left(\mathrm{R}_{E}\right)$, perigee of $1.8 \mathrm{R}_{E}$ and an inclination of $86^{\circ}$. Such an orbit allowed for measurements of the Earth's polar regions at both high- and low-altitudes in a single $18 \mathrm{~h}$ orbit. The satellite has good coverage of all MLTs over a period of 6 months, allowing for excellent, and even, data coverage over an extended period of time. Figure 1 illustrates every 30th Polar orbit between 16 May 1996 and 16 May 2002. Figure 1a shows the X-Y Geocentric Solar Magnetic (GSM) plane, and illustrates the spacecraft's magnetic local time coverage over the six year period from which data were analysed. The satellite's apogee was initially over the northern polar region and has precessed by approximately $18^{\circ}$ per year since orbit insertion, leading to an evolution in the altitudes at which both polar regions are being studied. Figure $1 \mathrm{~b}$ shows the GSM X-Z plane and illustrates the extent of the orbit precession, with apogee reaching the magnetic equator by May 2002. The satellite's orbit is fixed relative to the sun. In spring it traverses south to north in the daytime magnetosphere and vice-versa in autumn, this can be seen in Fig. 1b where the apogee of the orbit is located in the negative or positive $\mathrm{X}$ co-ordinate for spring and autumn respectively. The satellite has a $6 \mathrm{~s}$ period with the spin axis orthogonal to the plane of the orbit, and allows CAMMICE (MICS) to map out the complete $4 \pi$ steradian distribution function. The instrument measures particle energies over a large range, $1 \mathrm{keV}$ to $328 \mathrm{keV}$ (reduced from $448 \mathrm{keV}$ due to an instrument fault), utilising energy bins that are spaced logarithmically. The instrument has a potential mass range of 1 Amu to 55 Amu, allowing it to measure not only the protons that are dominant in the magnetosphere, but also heavier
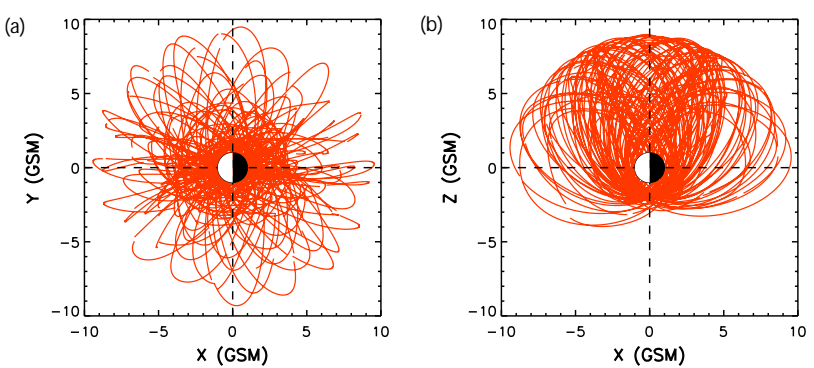

Fig. 1. Every 30th Polar orbit between 16 May 1996 and 16 May 2002 in Geocentric Solar Magnetic (GSM) co-ordinates. Panel (a) shows the GSM X-Y plane, and illustrates the magnetic local time coverage. Panel (b) shows the GSM X-Z plane, and illustrates the extent of the orbit precession towards the magnetic equator over the six year period.

particles such as oxygen ions. The full characterisation of incident particles is achieved by utilising a mixture of energy spectroscopy, time-of-flight analysis and electrostatic filtering to measure particle energy, mass and charge respectively. In addition, a narrow angle of acceptance allows for the determination of particle pitch angles.

\section{Statistical database}

The database comprises $\sim 7700$ IDFs from data recorded over a 6 year period between May 1996 and April 2002 when the Polar satellite was located between $60^{\circ}$ and $80^{\circ}$ invariant latitude. This region maps to the ionosphere over an area where many ground based instruments are in operation, such as EISCAT and the Troms $\varnothing$ high power radio frequency facility, the IMAGE, CANOPUS and other magnetometer networks, and SuperDARN. The data were collected using the Total $\mathrm{H}$ channel of the particle collectors, it was thus assumed that IDFs are dominated by protons to such an extent that contributions from other, heavier, ions were negligible. Firstly time intervals were extracted from the orbital data when Polar's ionospheric footprint, mapped using the Tsyganenko (1989) model into the northern hemisphere, was located at individual invariant latitudes, $x_{o}$, where each degree of invariant latitude is defined as $\left(x_{o}-0.5^{\circ}\right)-\left(x_{o}+0.5^{\circ}\right)$. Averaging the proton flux for each of the proton energies, as determined by CAMMICE (MICS) energy bins, over time from each single passage through a particular invariant latitude range created a data set of "invariant latitude averaged" IDFs. The data were then further binned according to hourly MLT; Fig. 2 presents the MLT coverage for a selection of invariant latitudes, and an excellent distribution is seen uniformly across all MLT bins (red bars).

To ensure that the rebinned, averaged IDF data had a sufficiently high count rate to allow a reliable average to be found, a limit on the minimum number of CAMMICE (MICS) time bins covered by the spacecraft over any particular invariant 
(a)
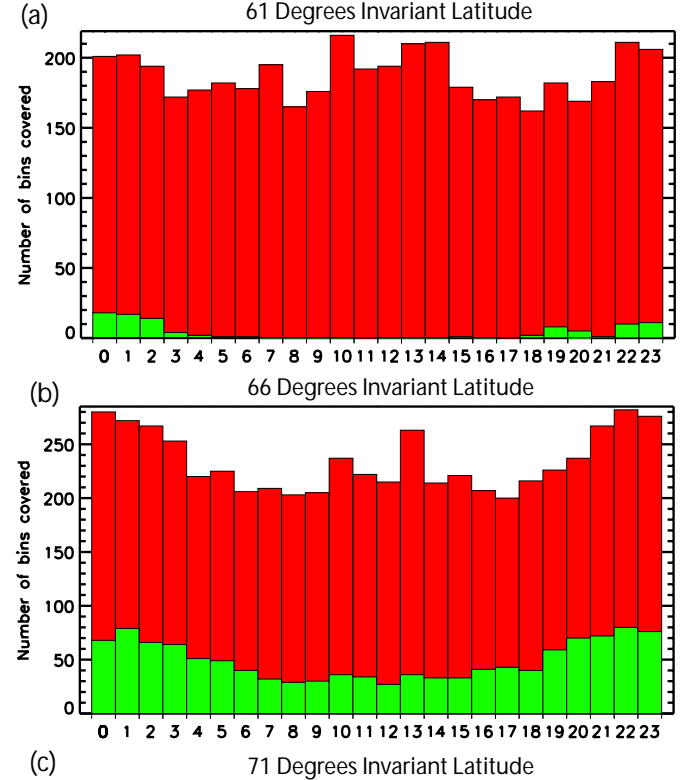

(c)

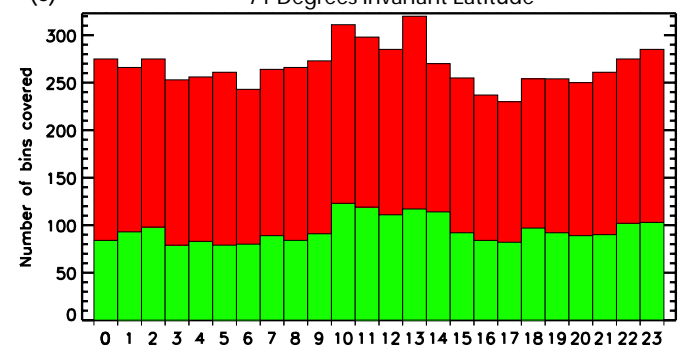

(d)

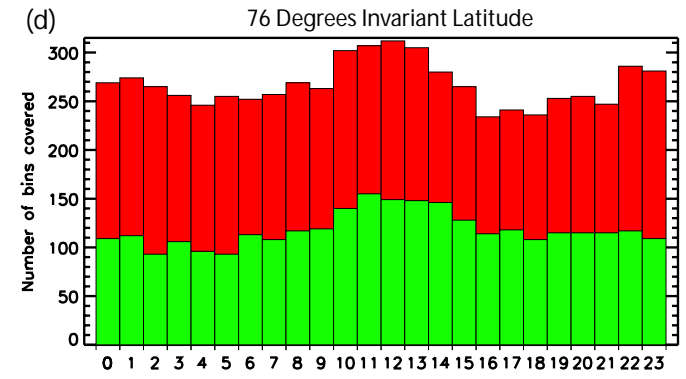

(e)

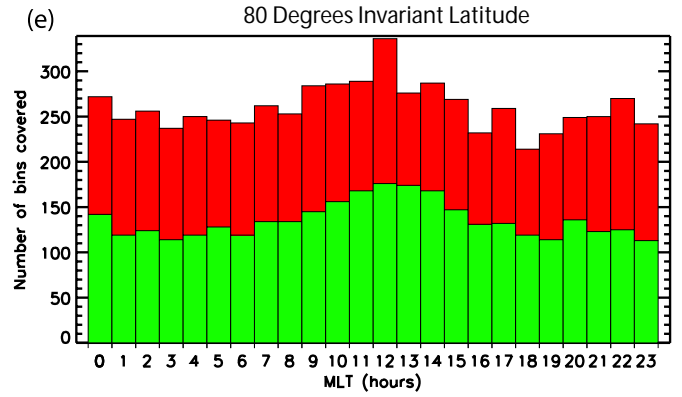

Fig. 2. Histograms for the number of Polar footprints in each MLT bin for select invariant latitudes. Red bars indicate the total number of footprints in each bin for the six years of CAMMICE (MICS) data being studied; green indicates those footprints that remain at that invariant latitude for at least five instrument time bins. latitude range was set. The coverage was set to at least 5 bins, equivalent to $\sim 16 \mathrm{~min}$, consistent with the study of Baddeley et al. (2004). This constraint reduced the viable data included in the database by $\sim 65 \%$ and is displayed for the sample invariant latitudes in Fig. 2 by the green bars. Polar's orbital orientation during its lifetime has resulted in the spacecraft traversing lower invariant latitudes with greater speed. A fixed range of invariant latitude also maps to a smaller volume in the magnetosphere at lower invariant latitudes. Both of these situations result in the spacecraft spending a shorter time in each individual invariant latitude bin for the lower latitudes, reducing the data set, as seen in Fig. 2a. Furthermore, particle distributions characteristic of open field lines were excluded from this study. This further reduction of viable data primarily removed data at the highest invariant latitudes, where open field lines are most common, and resulted in several invariant latitude bins being combined so that each MLT bin held enough invariant latitude averaged IDFs for reliable statistical calculations. Figure 3 illustrates the available data after both constraints discussed above have been applied and the invariant latitude bins have been combined where required. MLT bins highlighted in green have $\geq 50$ viable invariant latitude-averaged IDFs, enough for reliable statistical analysis; MLT bins in grey have $<50$ viable IDFs and so undergo no further analysis.

The final set of IDFs obtained was scanned for positive gradient regions. This required that there were 2 or more consecutive increases in particle flux with respect to increasing proton energies as defined by the 32 CAMMICE (MICS) energy bins. To prevent the identification of data spikes as positive gradient regions a criterion was set for the maximum magnitude of the change in $f(W)$ as $<1000 f(W)_{\min }$, where $f(W)$ and $f(W)_{\min }$ are the distribution function and the minimum value of the distribution function value for the positive gradient region of a particular IDF. Each positive gradient region identified had two attributes related to it recorded, the position of the positive gradient region in each distribution function, called the energy location, and the free energy contained in the region available to a wave. The energy locations were calculated from

$W_{a v}=\left(W_{\min }+W_{\max }\right) / 2$

where $W_{a v}$ is the average proton energy location, $W_{\min }$ is the minimum proton energy and $W_{\max }$ is the maximum proton energy in the positive gradient region. The free energy contained within each bump was calculated using the method described in Baddeley et al. (2004) using

$E_{\text {total }}=V * \Sigma_{i}\left(\partial n_{i}-\partial n_{\min }\right)\left(W_{i}-W_{\text {min }}\right)$

where $E_{\text {total }}$ is the amount of free energy available, $V$ is the volume of the dipole field flux tube the IDF is located in (here a cross sectional area of $1^{\circ}$ in latitude and $5^{\circ}$ in longitude, is used, as employed in Baddeley et al. (2004; 2005). This is a typical spatial extent for the wave events generated through 


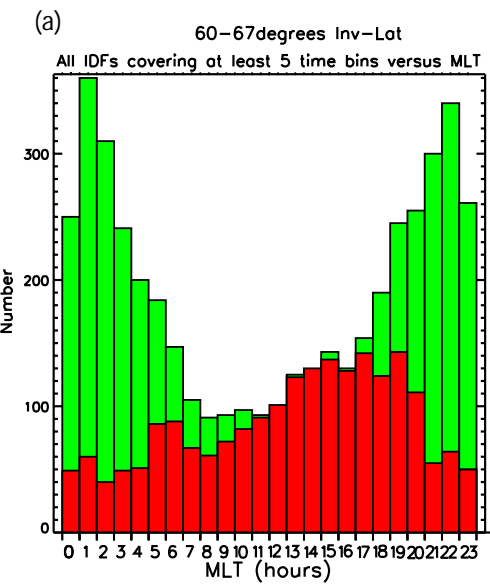

(d)

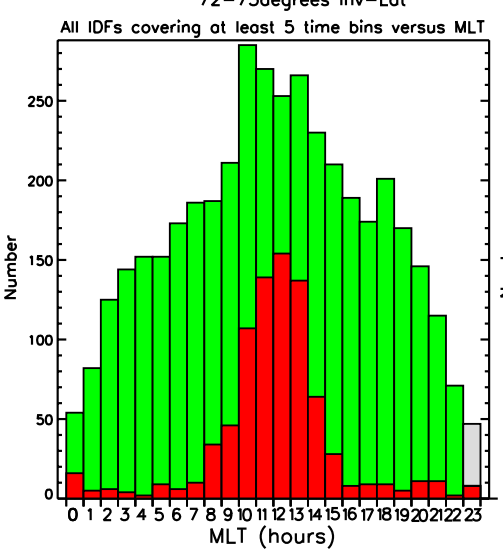

(b)

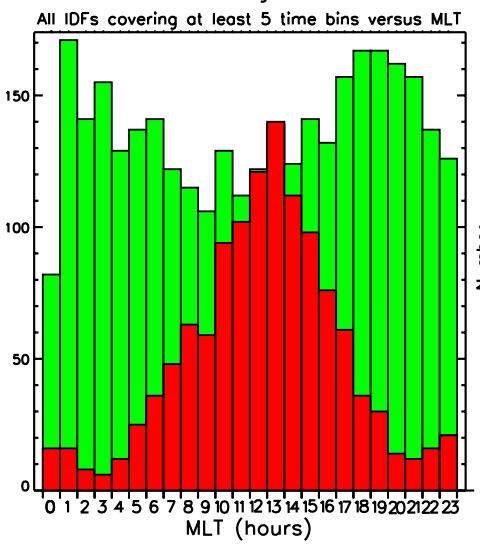

(e)

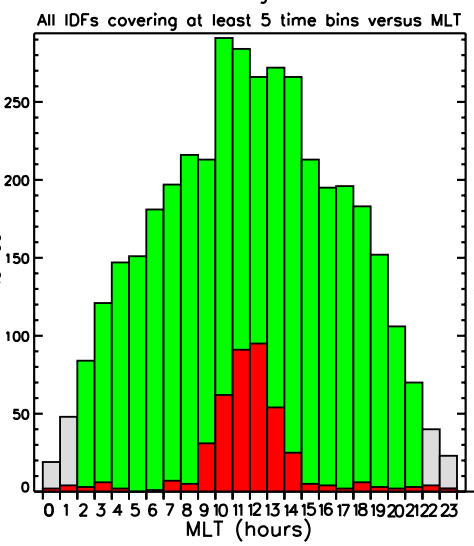

(c)

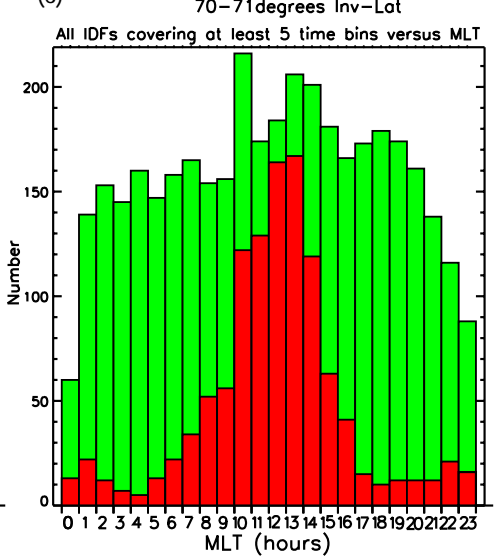

(f)

76-80degrees Inv-Lot

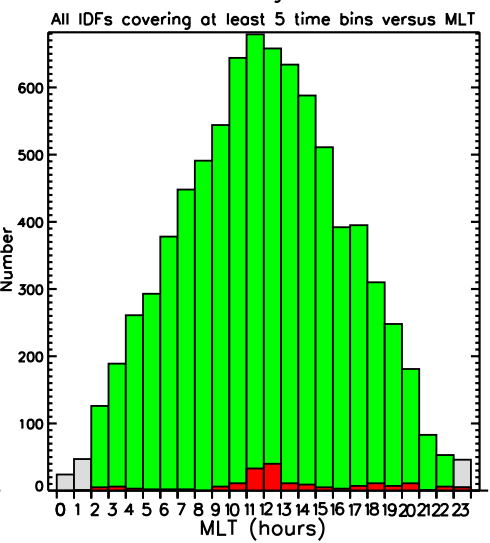

Fig. 3. Histograms showing all viable, observed ion distribution functions in each MLT bin for various invariant latitude ranges. Grey bars indicate MLT bins where there are less than 50 such IDFs available for statistical analysis. The red bars indicate the number of IDFs in each MLT bin which were characterised by a positive gradient.

wave particle interactions, $\partial n_{i}$ is the average number density of particles at the average energy of a CAMMICE (MICS) energy bin $W_{i}$, and $\partial n_{\min }$ is the number density of particles with energy $W_{\min }$. Figure 4 illustrates a typical IDF with a bump feature; the key values are labelled, and in this case a total free energy of $2.43 \times 10^{10} \mathrm{~J}$ was calculated from Eq. (4) above as described in Baddeley et al. (2004). A number of IDFs recorded contain multiple positive gradient regions, in these cases each region is considered separately as long as they each meet the criteria specified above.

Ion distribution functions identified as containing positive gradient regions are indicated in Fig. 3 by the red bars. At the lowest latitudes, Fig. 3a, positive gradient regions are more populous in the afternoon MLTs, whilst at higher latitudes the occurrence of such regions form more symmetrical distributions centred at $\sim 12: 00$ MLT. As latitude increases, the total number of positive gradient regions for all MLTs decreases, but do so more rapidly the further from noon they occur. In panels a and b of Fig. 3, for MLTs 13:00 and 14:00 respectively, the number of positive gradient regions identified are actually greater than the total number of IDFs for these particular MLTs (shown by the green bars as previously mentioned). This results from there being a significant number of IDFs containing more than one positive gradient region.

\section{Statistical analysis}

Initially, when identifying positive gradient regions in the IDF data there was no limit set on the magnitude of the free energy contained in the positive gradient regions in order to examine the full range of free energies available. Figure 5 shows the variation of the mean, median and the quartile ranges of all free energies with respect to invariant latitude over the $60^{\circ}-80^{\circ}$ range, averaging over all proton energies and MLT locations. A consequence of this approach is that data can be represented for each individual 1 degree invariant latitude bin as there are enough IDFs per invariant latitude for a viable statistical analysis. The number of IDFs used in each bin is indicated by the red line, with the scale shown on the right hand side. Figure 5 shows that the free energy available 


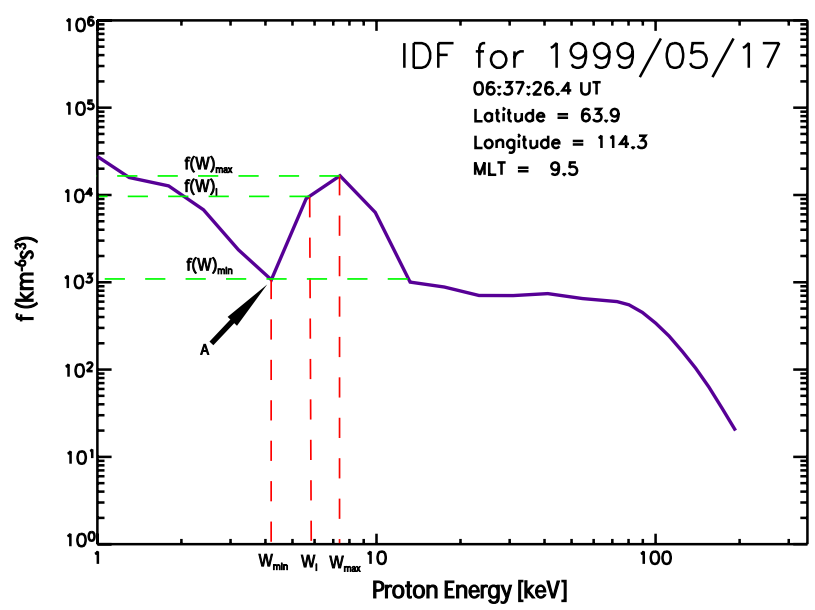

Fig. 4. An example of an IDF containing a positive gradient region. The number densities $\partial n_{\min }$ and $\partial n_{i}$ from Eq. (4) are calculated from the values $f(W)_{\min }$ and $f(W)_{i}$, respectively, where $W$ is the proton energy. All data points located on the positive gradient, i.e. those between $W_{\min }$ and $W_{\max }$, transpose to the location in phase space marked as point $\mathrm{A}$.

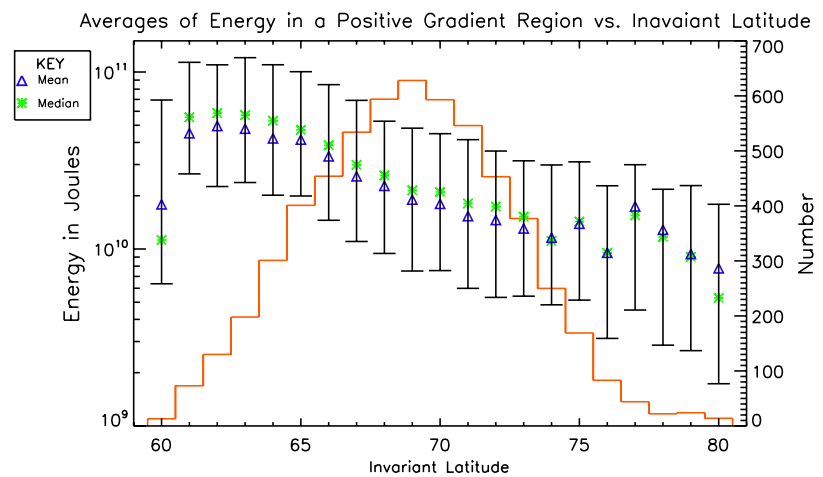

Fig. 5. The variation of mean and median free energy in Joules contained in positive gradient regions with invariant latitude. Quartiles are displayed to give an indication of data spread. The number of IDFs used in each bin is indicated by the red line histogram, with the scale shown on the right hand side.

in positive gradient regions is typically $\sim 10^{10} \mathrm{~J}$, also revealed is the decrease of the average energy with increasing invariant latitude. Over the $20^{\circ}$ range in invariant latitude the amount of free energy drops by an order of magnitude, and there is good agreement between the mean and median, indicating a small skewness in the distribution of energies. The quartiles, representing the spread of the middle $50 \%$ of all data, decrease by a factor of $\sim 5$ as latitude increases, taking in to account that the energy scale is logarithmic. Finally $>75 \%$ of the data have $>10^{9} \mathrm{~J}$ of free energy for all invariant latitudes. This provides three natural free energy limits to work with; $>1 \times 10^{9} \mathrm{~J},>1 \times 10^{10} \mathrm{~J}$ and $>1 \times 10^{11} \mathrm{~J}$, representing the majority of the data, a typical reference level and an upper level to look at the few highest free energy positive gradient regions, respectively. These free energy thresholds are used in the analysis of the MLT variation of the proton energy of the positive gradient regions discussed next.

As with the earlier study by Baddeley et al. (2004) the results are displayed in a series of "clock plots". Figures 6, 7 and 8 show the percentage rates of occurrence for IDFs with positive gradient regions binned by proton energy and MLT, compared to the total invariant latitude-averaged IDFs in any particular MLT bin; where Fig. 6 includes all IDFs containing a free energy of $>1 \times 10^{9} \mathrm{~J}$; Fig. $7>1 \times 10^{10} \mathrm{~J}$ and Fig. 8 $>1 \times 10^{11} \mathrm{~J}$. In each clock plot the radial distance outwards is the energy location for the protons in the positive gradient region. The data has been binned according to the CAMMICE (MICS) energy bins. The MLT is indicated by the angular location, with midnight to the bottom, noon to the top, dawn to the right and dusk to the left. Figures 6,7 and 8 contain six panels, a-f, each one representing data from a group of invariant latitudes; the greyed out MLT bins in panels $\mathrm{d}-\mathrm{f}$ are equivalent to those greyed out in Fig. 3 and are the result of there not being enough IDFs to provide a reliable analysis. The colour scale is saturated at $24 \%$ in order to emphasise the variations in occurrence at lower percentage values.

At the lowest invariant latitudes, Fig. 6a, the highest occurrence rates, 32\%-50\%, are located between 13:00 MLT and 15:00 MLT reaching a peak at proton energies $\sim 4 \mathrm{keV}$ to $\sim 8 \mathrm{keV}$. From 15:00 MLT through the dusk sector to 21:00 MLT the focus is still in this proton energy range, although occurrences have dropped to $<\sim 30 \%$. At higher proton energies from 12:00 MLT to 21:00 MLT there is a scattering of occurrences up to $\sim 250 \mathrm{keV}$ (here the maximum proton energy displayed is $100 \mathrm{keV}$ for clarity of the major features) and below $5 \mathrm{keV}$. In the midnight sector, 21:00 MLT to 01:00 MLT, the entire range of proton energies are covered with low occurrence rates, it is relatively unlikely that a wave particle interaction will be observed in this region when compared to the afternoon sector. For the whole morning sector, 01:00 MLT to 13:00 MLT, occurrence rates peak at $>\sim 10 \%$ around $\sim 7 \mathrm{keV}$ with a slight occurrence rate increase at 10:00 MLT. Similarly there is a second peak in the proton energy at $\sim 20 \mathrm{keV}$ to $30 \mathrm{keV}$ in the morning sector, but with slightly lower rates of occurrence. Over higher latitude ranges, see Figs. $6 \mathrm{~b}-\mathrm{c}$, this second, higher energy peak is not evident.

Over the $68^{\circ}-69^{\circ}$ invariant latitude range, Fig. $6 \mathrm{~b}$, positive gradients located at $>10 \mathrm{keV}$ are considerably less prevalent, except in the morning sector, 04:00 to 12:00 MLT, here they still occur out to $\sim 40 \mathrm{keV}$ with the highest rate of occurrence at $7 \mathrm{keV}$. This signifies a general shift in the overall occurrence rate towards the dawn sector over the operational latitude of the Troms $\emptyset$ high power radar facility used in many ULF wave studies. Moving round to the location 12:00 to 15:00 MLT, the high proton energy locations fall away, but there are still significant numbers, $\sim 36 \%$ occurrence, at $\sim 5 \mathrm{keV}$ as also seen in Fig. $6 \mathrm{a}$. The dusk sector at 

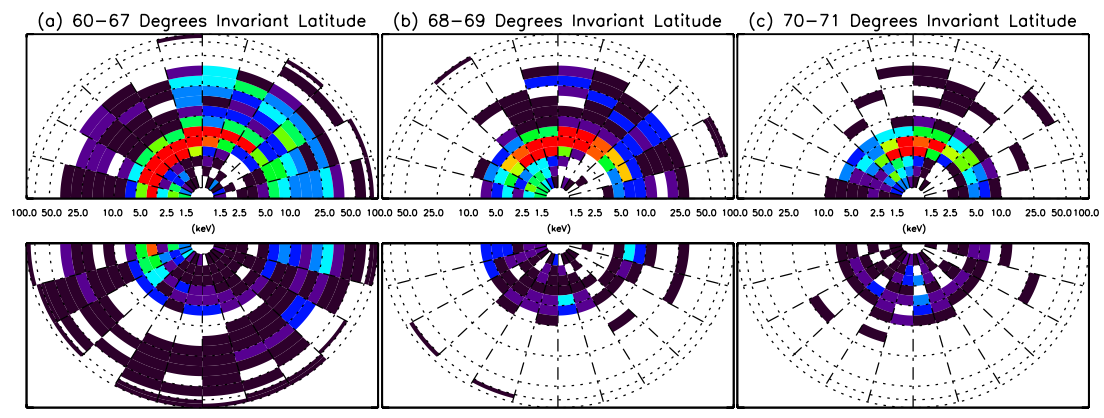

Positive Gradient Regions Containing 1.0e+09 J Or Greater
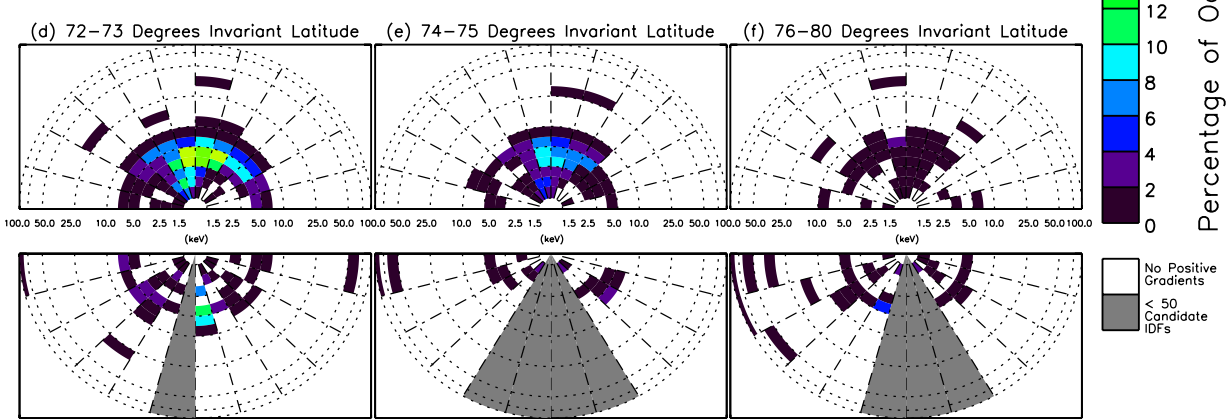

Fig. 6. The occurrence of IDF positive gradients containing $>10^{9} \mathrm{~J}$ of free energy resulting from the statistical study of the magnetospheric particle population data gathered using the CAMMICE (MICS) instrument. The six years of data has been grouped by invariant latitude in panels (a) through (f); each panel is binned by MLT, shown by angular location with midnight to the bottom moving anti-clockwise through dawn, noon and dusk. Proton energy location, binned according to CAMMICE's energy bins is represented by the radial position. Grey MLT bins indicate regions containing too few IDFs for reliable analysis.

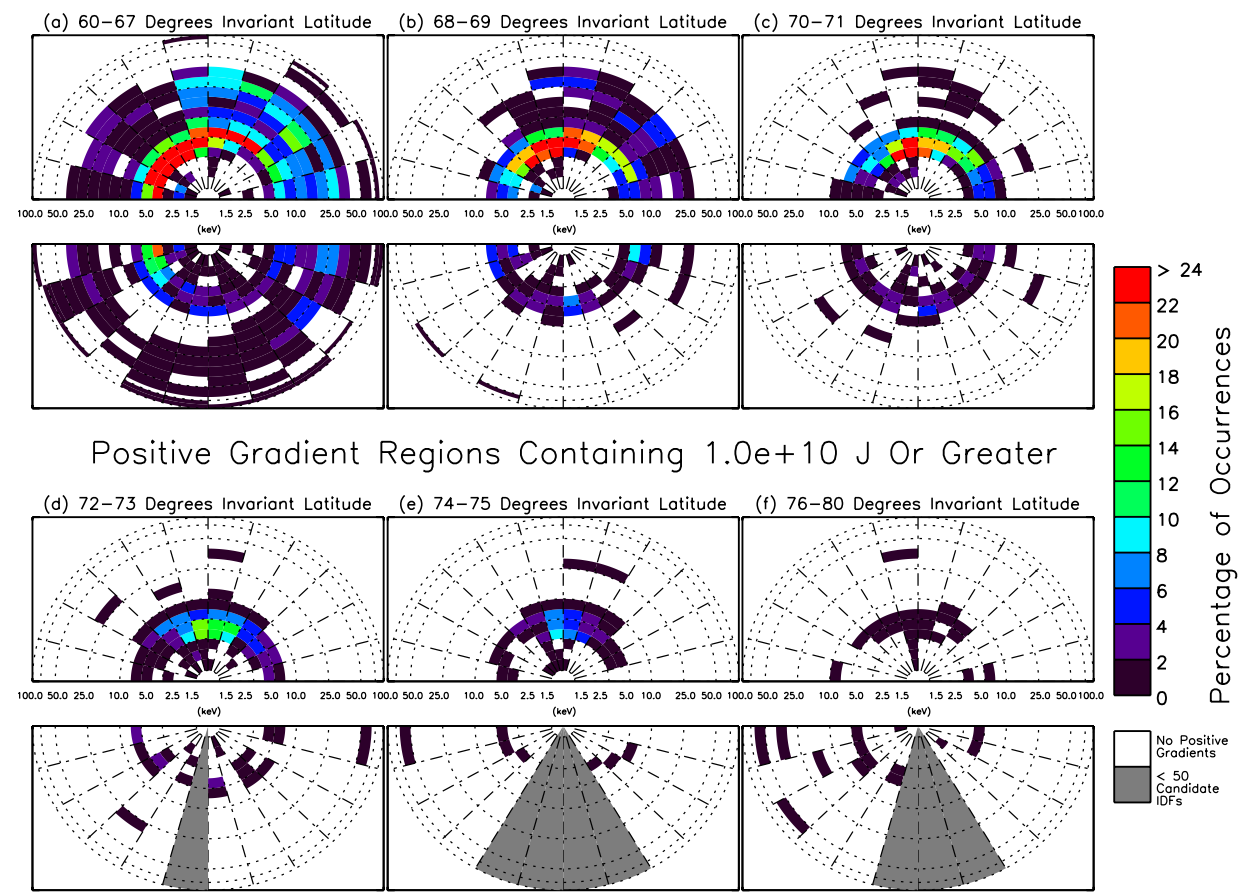

Fig. 7. As Fig. 6 except all panels show the percentage occurrence of IDFs with positive gradient regions containing $>10^{10} \mathrm{~J}$ of free energy. 


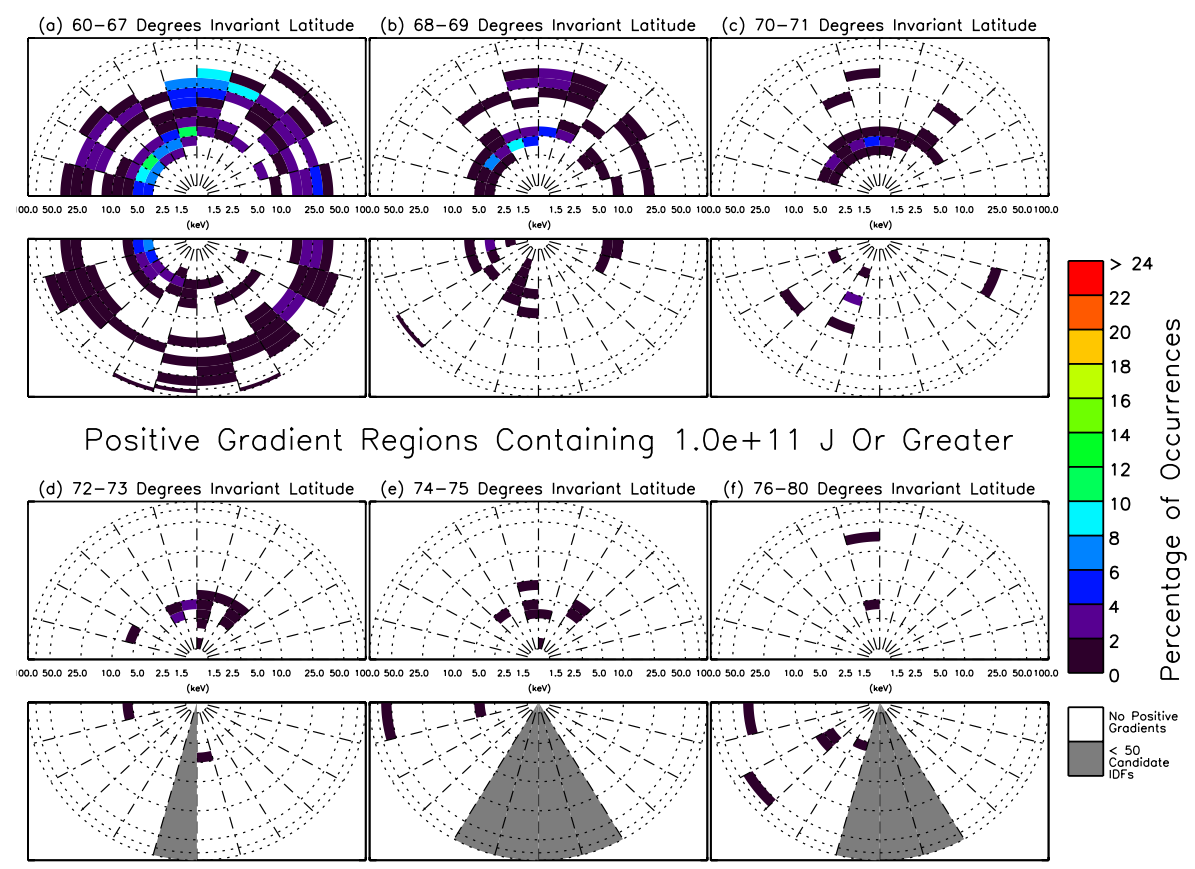

Fig. 8. As Fig. 6 except all panels show the percentage occurrence of IDFs with positive gradient regions containing $>10^{11} \mathrm{~J}$ of free energy.

these invariant latitudes is now sparsely populated by IDFs with positive gradients, as is the midnight sector, indicating a reduction in the possibilities of wave generation over these MLTs. At higher invariant latitudes of $70^{\circ}-71^{\circ}$, Fig. $6 \mathrm{c}$ there is further reduction in positive gradients at higher proton energy locations including those in the dawn sector. The main focus of occurrences is still located at $\sim 5 \mathrm{keV}$ to $\sim 10 \mathrm{keV}$ between the hours of 08:00 MLT to 14:00 MLT.

Increasing invariant latitude even further, the results presented in Figs. 6d, e and $\mathrm{f}$ show a trend of reduced occurrence rates at high proton energies with the focus remaining on the morning sector close to noon. The greyed out MLT bins in the midnight sector are the result of there being $<50$ original IDFs that could possibly contain positive gradient regions. It should be noted that at these higher invariant latitudes the open-closed magnetic field line boundary (OCFLB) has an effect on the results. Open field lines can not support IDFs with positive gradient regions as they can no longer trap particles in the magnetosphere. The OCFLB is located such that in the midnight sector it is at lower invariant latitudes than it is in the midday sector, and this will be discussed later in this section.

Figure 6 employs a lower limit of $1 \times 10^{9} \mathrm{~J}$ as an energy threshold for identifying positive gradient regions. Only $\sim 10 \%$ of all identified IDFs with positive gradient regions have a free energy of less than this, so Fig. 6 is a good representation of all the observed positive gradient regions. Figures 7 and 8 present the data in the same format, but with a progressively higher energy threshold and thus present data with high enough free energies to drive ULF waves with sig- nificant amplitudes (Baddeley et al., 2004). Comparing the panels in Fig. 7 directly to the corresponding ones in Fig. 6 there is only an $\sim 5 \%$ difference in the positive gradient occurrence rate for all MLTs; most IDFs have $>10^{10} \mathrm{~J}$ available. Figure 8 , however, indicates that few extend to energies as high as $10^{11} \mathrm{~J}$, with an $\sim 90 \%$ difference when compared to Fig. 7. The largest calculated free energy value is $7.1 \times 10^{12} \mathrm{~J}$. Of all the data recorded $\sim 75 \%$ of positive gradient regions contain between $10^{10} \mathrm{~J}$ and $10^{11} \mathrm{~J}$ of free energy. Under all three energy thresholds the prevalence of the positive gradient region is on the day side, although it is less clear in Fig. 8a where there is an almost even distribution over all MLTs, but at low occurrence rates. Furthermore, the low proton energy peak, seen in panel a of Figs. 6 and 7, in the morning sector is not present in Fig. 8a, whilst the high proton energy peak remains in evidence. This indicates that the higher proton energy positive gradient regions are those that contain the largest amounts of free energy for the morning sector.

Figures 7 and 8 show a greater loss of positive gradient region occurrences in nightside MLTs compared to those closer to noon as invariant latitude increases. Figure 9 presents the OCFLB and was generated, using the same six years of CAMMICE (MICS) data used in creating the IDF database, by scanning the data for events where Polar moved from a particle population indicative of closed field lines to that of open field lines or vice-versa. When Polar moved from being on a closed field line CAMMICE (MICS) registered a reduction of proton flux as the open field lines have no trapped particle populations. The distribution of the average invariant 
latitude of Polar's footprint where this occurred has been binned by MLT. The result is that closed field lines are often available at invariant latitudes up to almost $80^{\circ}$ at noon, but are rarely present at this latitude for 20:00 through to 04:00 MLT, explaining the lower high-latitude occurrence rates of IDFs at nightside MLTs depicted in Figs. 6-8.

At lower invariant latitudes, waves generated by waveparticle interactions might be expected to grow to larger amplitudes, and consequently dissipate more energy into the ionosphere, than those at higher invariant latitudes as the IDF positive gradient regions contain larger free energies there. However, in instances of higher amounts of available energy, the drift-bounce resonance condition (Eq. 2) is also required to be satisfied for wave growth to occur, and this depends critically on the energy location of the positive gradient region.

Figure 10 presents the variation of the mean, median and mode of the proton energy, $W_{a v}$, location as a function of invariant latitude. The number of IDFs used in each bin is indicated by the red line, with the scale shown on the right hand side. The mode is fairly constant at $\sim 4 \mathrm{keV}$ over the entire invariant latitude range, so the most common proton energy at which positive gradient regions occur is largely independent of invariant latitude. Over the middle latitudes of the range, the median and mean are close to the mode, and the quartiles indicate that the distributions are narrow and evenly spread. At the lower invariant latitudes the median departs towards higher proton energies than the mode, as does the mean, but more dramatically. To maintain the detail in the separation of the averages in Fig. 10 the proton energy scale has an upper limit of only $25 \mathrm{keV}$. This does, however, lead to the upper quartile values for $60^{\circ}, 61^{\circ}$ and $80^{\circ}$ being absent from the plot. The separation of the mean, median and mode is due to the double peak seen in the proton energy occurrence that is observed in the morning sector at the lower invariant latitudes. This is further highlighted by the greater quartile ranges. The mode of the data reveals that low proton energy positive gradient regions dominate in number, however, the high proton energy peak $(\sim 20 \mathrm{keV}-30 \mathrm{keV})$ is significant within the population at low latitudes. At least $25 \%$ of the data, as illustrated by the quartile ranges, are above $20 \mathrm{keV}$ for the lowest invariant latitudes. In contrast, for latitudes $66^{\circ}-76^{\circ}$, where the higher energy peak in occurrence rate is not evident, see Fig. 8, 75\% of the data is below $\sim 6 \mathrm{keV}$. At the extreme upper and lower ends of the latitude scale there is further separation of the mean, median and mode. In this instance, however, it is likely due to the low number of IDFs present, leading to spurious results.

Figure 11 illustrates the mean, median and mode proton energy location as a function of MLT. The number of IDFs used in each bin is indicated by the red line, with the scale shown on the right hand side. There is fairly close agreement between the mean, median and mode at 11:00-17:00 MLT, indicating a single peak in the distribution of the positive gradient regions centred at $\sim 4-5 \mathrm{keV}$. Across the dawn sec-

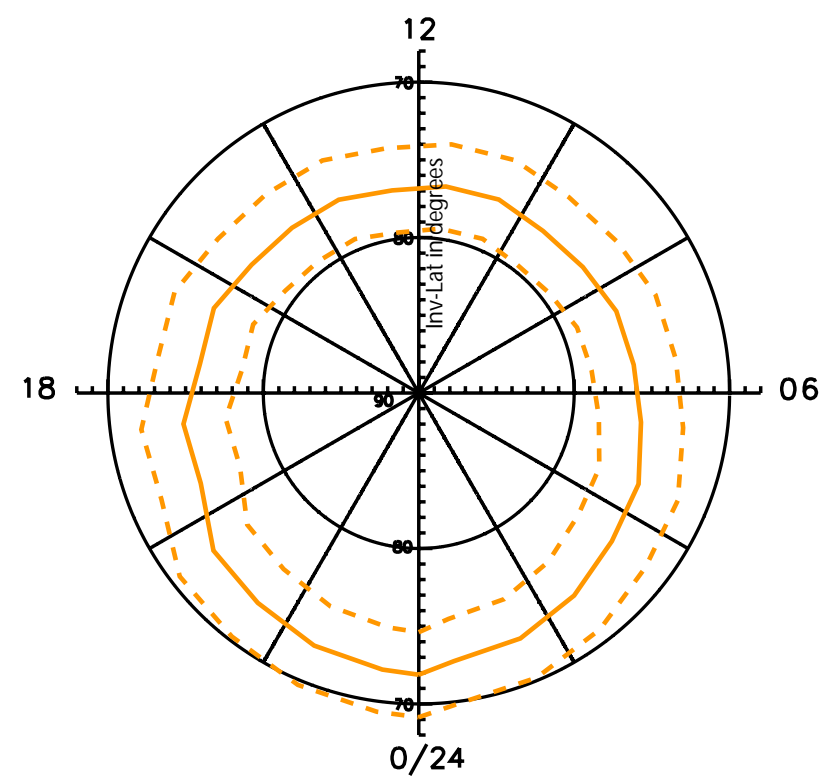

Fig. 9. The open-closed magnetic field line boundary in the northern hemisphere as identified using a statistical analysis of the six years of CAMMICE (MICS) data. MLT is defined by angular position with midnight at the bottom and moving anti-clockwise through dawn noon and dusk; invariant latitude is radial. The solid orange line is the mean boundary location over all six years of data with the dashed orange line indicating the boundary at \pm 1 standard deviation form the mean.

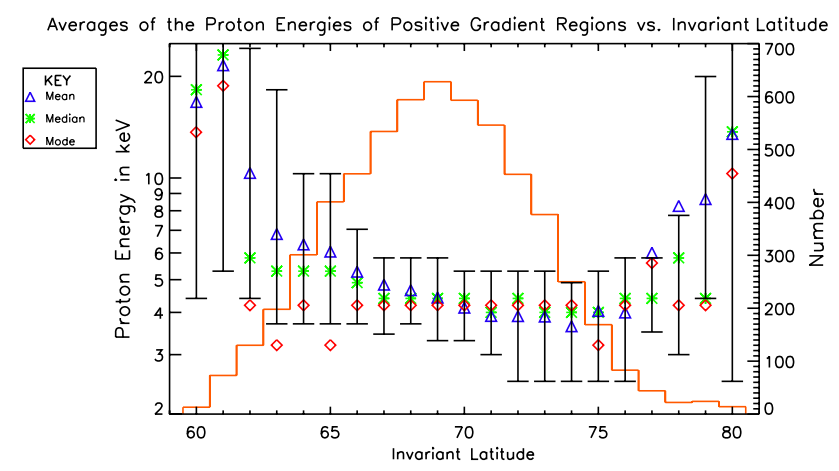

Fig. 10. The variation of the mean, median and mode of the proton energy location with respect to invariant latitude. Quartiles are displayed to give an indication of data spread. The number of IDFs used in each bin is indicated by the red line histogram, with the scale shown on the right hand side.

tor the agreement between the mean, median and mode diminishes where the double peak in the occurrence rate is observed in the morning sector with the mean extending up to $\sim 10 \mathrm{keV}$ at 05:00 MLT. Furthermore, the spread of data in the dawn sector, shown with quartiles, extends up to much higher proton energies when compared to any other times. This can particularly be seen at 05:00 MLT where at least 


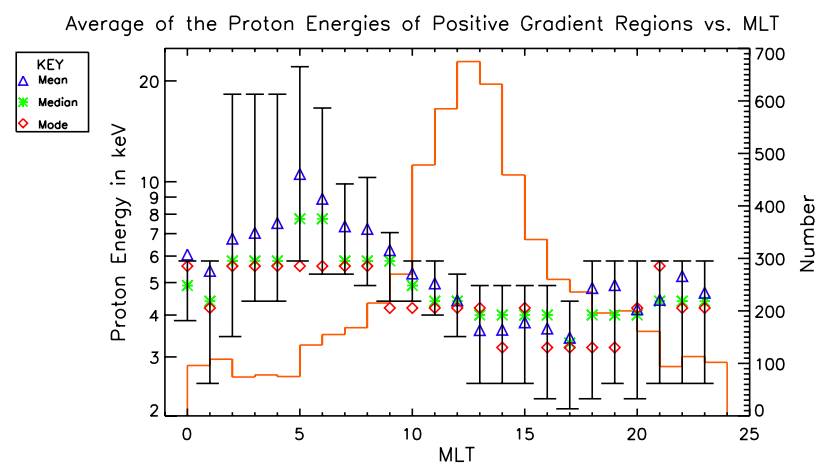

Fig. 11. The variation of the mean, median and mode of the proton energy location with respect to MLT location. Quartiles are displayed to give an indication of data spread. The number of IDFs used in each bin is indicated by the red line histogram, with the scale shown on the right hand side.

$25 \%$ of the data are above $20 \mathrm{keV}$ compared to, for example, 18:00 MLT where $25 \%$ of the data lie above $\sim 6 \mathrm{keV}$. The spread of proton energies for the occurrence of positive gradient regions minimises at 10:00 MLT.

\section{Discussion}

Previously Baddeley et al. (2004) conducted a similar study to that presented here. Using data collected over 2.5 years from both CAMMICE (MICS) and TIMAS instruments on Polar, unstable ion populations between, and including, invariant latitudes $67^{\circ}$ to $70^{\circ}$ were analysed. They concluded that lower energy $(10-45 \mathrm{keV})$ protons are the dominant unstable, non-Maxwellian populations over that invariant latitude range with free energies of typically $>10^{10} \mathrm{~J}$. The free energy associated with higher energy protons $(>100 \mathrm{keV})$ was found to be, in general, at least an order of magnitude less and typically $<10^{9} \mathrm{~J}$. Furthermore, they concluded that the majority of unstable IDFs occurred in the morning sector between 05:00 and 12:00 MLT thus making it a fertile ground for high $m$ ULF wave generation. They also concluded that Pgs (see e.g. Chisham and Orr, 1991) are more likely to be even mode events driven by a drift-bounce resonance interaction $(N=1$ in Eq. 2).

Particle populations which display unstable, nonMaxwellian distribution functions, provide energy for high $m$ ULF waves through the process of inverse Landau damping. Here the analysis of ion populations in the magnetospheric ring current is extended to explore the expected latitudinal variation of the occurrence of such waves. Using 6 years of particle data gathered from the CAMMICE (MICS) particle detector covering invariant latitudes $60^{\circ}-80^{\circ}$ the prevalence of ion populations has again been scrutinised. Using this larger data set it has been possible to draw conclusions on the variation in the occurrence of IDFs with positive gradient regions with respect to invariant latitude, MLT location and free energy. However, there still remains uncertainty in the possible role of spatial gradients in IDFs (the right-hand term in Eq. 1) since Polar is only a single satellite; this does not, however, detract from the conclusions of this paper.

Overall the results presented here are in good agreement with the previous study undertaken by Baddeley et al. (2004). Figures 5, 6, 7 and 8 show that the free energy available in unstable IDFs is largely in the $10^{9} \mathrm{~J}-10^{11} \mathrm{~J}$ range. This is consistent with previous observations of internally driven ULF waves through inverse Landau damping. Allan and Poulter (1984) reported observations of two particle driven waves; a Pc5 pulsation dissipating $\sim 10^{10} \mathrm{~J}$ and a Giant Pulsation $(\mathrm{Pg})$ dissipating $\sim 10^{11} \mathrm{~J}$ into the ionosphere, both consistent with the findings here. We will discuss implications for wave generation at various MLTs and invariant latitudes below.

5.1 The variation of the MLT distribution with invariant latitude

The evolution in the MLT occurrence of bump-on-tail IDFs with increasing invariant latitude can be seen in Figs. 6, 7 and, to a lesser extent, 8 progressing from panel a to $f$ in each case. The percentage occurrence of IDFs with positive gradient regions decreases, from highly populous to rather sparse as invariant latitude increases over the $20^{\circ}$ range under investigation. For the lowest invariant latitudes $\left(\leq 68^{\circ}\right.$, see panel a of Figs. 6, 7 and 8) the highest rates of occurrence cover from 10:00 to 19:00 MLT, typically with protons of energies $\sim<10 \mathrm{keV}$, while in the morning sector there is a twin peak in the occurrence rate at $\sim 8 \mathrm{keV}$ and $\sim 20$ $30 \mathrm{keV}$. Ground based observations of storm time Pc5s, observed in the afternoon sector, have previously been undertaken (e.g. Allan et al., 1982, 1983) and drift resonance between odd mode standing structures and particles of energies $\sim 10-70 \mathrm{keV}$ were considered to be the most likely cause of these events. The lowest values of this range are in near agreement with the findings of this study. Furthermore, several spacecraft studies have also studied wave events in the afternoon sector of the magnetosphere at these invariant latitudes (e.g. Anderson, 1993; Anderson et al., 1990; Lessard et al., 1999; Woch et al., 1990) and have drawn similar conclusions on the energy of the driving particle populations.

Ozeke and Mann (2001) conducted modelling work on the drift path trajectories of magnetospheric protons in a dipole field; they found that the model accommodated solutions for both storm time Pc5s, and radially polarised Pc4 pulsations in the afternoon sector. Applying an odd mode standing wave solution $(N=0)$ in Eq. (2) indicated favourable conditions over a range of $L$-shells for varying mnumbers, $L \sim 6.5$ for $m=-30$ and $L \sim 5.1$ for $m=-100$. The locations in $L$ space are comparable to the low invariant latitude $\left(<65^{\circ}\right)$ locations of bump-on-tail occurrences in this study where the afternoon sector is dominant over the morning sector. Their 
conclusions for Pc4 waves in the afternoon sector were, however, more reserved; finding the $L$ space location to be limited to $L \sim 3.2$, lower than the invariant latitudes covered in this study.

Prior statistical studies on high $m$ ULF waves using satellite data, while agreeing with the conditions in which afternoon sector wave events occur, yield dissimilar conclusions to this study and some ground based studies (e.g. Yeoman et al., 2000) as to the predominant MLT of the observed waves. They imply that the dusk sector is the more prevalent region for the generation of ULF waves. The present study, along with Baddeley et al. (2004) and Yeoman et al. (2000) have an advantage over the earlier studies due to the substantial databases of measurements employed and together make a strong case for a higher occurrence rate to be likely in the morning sector at higher invariant latitudes (see below).

As invariant latitude increases the predominant region of bump-on-tail IDFs is found to move to the noon sector, as shown in panels $b$ through $d$ in Figs. 6 and 7. This is in keeping with the findings of Baddeley et al. (2004) for the invariant latitudes of $67^{\circ}-70^{\circ}$. With this shift it is expected that wave generation in the morning sector becomes increasingly likely with higher invariant latitudes up to $\sim 70^{\circ}$. At higher latitudes it is increasingly likely that the OCFLB will be reached, and so trapped particle populations are less likely to be available to allow wave particle interactions of the type of interest to this paper except close to noon MLT. This is demonstrated in panels e and f for Figs. 6, 7 and 8, as the occurrence rate drops off rapidly at the higher invariant latitudes starting at first in the midnight sector and progressing through dawn and dusk towards noon. Further agreement is found with the work by Yeoman et al. (2000) which found a strong pre-noon dominance of high $m$ ULF wave activity utilising a HF Doppler sounder (DOPE). They found a total of 41 out of 52 events were observed in morning sector MLTs that were uncorrelated with magnetometer observations, with most waves observed between 06:00-10:00 MLT, in keeping with this study. Chisham and Orr (1991) also previously reported, in a study of $34 \mathrm{Pgs}$, that occurrence was in the morning sector between 02:00 and 09:00 MLT, peaking at 03:00-06:00 MLT. The authors found that the events were limited to $L$-shells 5.75-7.75, corresponding to the middle of the invariant latitude range of this study $\left(66^{\circ}-68^{\circ}\right)$. These observations are in keeping with this study under the assumption that Pgs need $>10^{11} \mathrm{~J}$ of energy, supplied by protons of $\sim 20-30 \mathrm{keV}$, which are seen throughout the morning sector at the lower latitudes, see Fig. 8a. This supports further the modelling work by Ozeke and Mann (2001). Pgs in the morning sector were concluded to be the result of a resonance interaction during a quiet time magnetosphere by Chisham (1996); allowing the particle populations to drift westwards round to the morning sector as the $\boldsymbol{E} \times \boldsymbol{B}$ drift is small enough to leave them trapped. Using these favourable conditions Ozeke and Mann (2001) calculated the drift path trajectories of magnetospheric protons in a dipole magnetic field in the morning sector. This revealed favourable locations in particle energy and $L$-shell space ( $W$ - $L$ space) for high $m$ wave growth to occur. In contrast to the afternoon sector results discussed above, their model implied the existence of highly monochromatic Pc4 waves that were localised to latitudes in the auroral zone $(L=6-10)$ as a result of drift-bounce resonance interactions between an even mode standing mode wave and $\sim 10 \mathrm{keV}$ protons. This information is in agreement with this study, where above $70^{\circ}$ invariant latitude the number of bump-on-tail features in the ion distributions falls off rapidly.

Thompson et al. (2001) linked observations of a Pg to an odd mode wave driven by a proton population at 100 $150 \mathrm{keV}$. Using proton fluxes from the geosynchronous Los Alamos National Laboratory (LANL) satellite they noted a fluctuation in the $125-153 \mathrm{keV}$ channel with a frequency matching that of their observed odd mode Pg wave. However, this is not in itself evidence that protons of these energies were driving the wave and is not in agreement with the work presented here. While it may be that a wave in the magnetic field was being modulated by this fluctuation of the proton flux other options cannot be ruled out without measurements of the particle distribution function. Baumjohann et al. (1987) observed a similar proton flux fluctuation in the AMPTE/IRM satellite whilst investigating a Pc5 wave event, and noted a positive gradient region located between energies of $\sim 1-30 \mathrm{keV}$. The ultimate conclusion was the possibility that the odd mode compressional pulsation was driven through a process of drift resonance such as a drift mirror mode (e.g. Pokhotelov et al., 1986). This study also finds the Thompson et al. (2001) wave event to be unlikely to result from a population inversion at such high proton energies, as all bump-on-tail distribution functions located at such proton energies do not harbour sufficient free energy to provide wave growth in Pgs (see Allan and Poulter, 1984). It is, therefore, suggested that, like Baumjohann et al. (1987), protons of energies $\sim 20-30 \mathrm{keV}$, as identified in this study, are responsible for the Pg wave event seen in Thompson et al. (2001) and led the modulation of proton fluxes observed at higher population energies. Thus the mode of the Thompson et al. (2001) event may have been misidentified, or alternatively it may have arisen from spatial gradients in the distribution function, as described in Eq. (1).

\subsection{Free energy variation with invariant latitude}

The free energy in a positive gradient region of an IDF is also found to vary with invariant latitude. As the observed invariant latitude increases the free energy decreases by an order of magnitude over the $20^{\circ}$ range. At higher invariant latitudes $\left(\geq 72^{\circ}\right)$ the free energy has dropped significantly, but, at the same time, the particle location becomes restricted almost exclusively to $<40 \mathrm{keV}$. Pg wave generation at high invariant latitudes is much less likely as their large amplitude requires larger energies, $\sim 10^{11} \mathrm{~J}$, while Pc5 events require less free 
energy to be available to them, $\sim 10^{10} \mathrm{~J}$ (Allan and Poulter, 1984). This indicates Pc5 generation may continue to be seen for a wide range of invariant latitudes up to $\sim 73^{\circ}$ where occurrence rates of the required ion distributions decrease.

\subsection{Proton energy variation with invariant latitude and MLT}

The proton energy of positive gradient regions is affected by both invariant latitude and MLT position (see Figs. 10 and 11). As invariant latitude is increased positive gradient regions at $>40 \mathrm{keV}$ rapidly disappear (see panels a-c of Figs. 6 and 7 in particular), however, positive gradient regions at $\leq 10 \mathrm{keV}$ are dominant more uniformly with invariant latitude in comparison to other proton energies, as revealed in Figs. 6, 7 and 10. Furthermore, the proton energy location is seen to vary with MLT to the extent that while the afternoon MLTs lose all positive gradient regions in the IDFs fairly uniformly over the proton energy range $>10 \mathrm{keV}$ as invariant latitude increases, the morning sector does not. This leads to a larger spread in proton energies making it more likely that the drift bounce resonance condition (Eq. 2) can be satisfied. This is interesting, as it remains uncertain what the proton energies of the bump-on-tail features in the driving particle populations are for morning sector ULF waves, in part due to there being no clear answer to the harmonic mode of Pgs. However, the higher energy ions proposed are uncommonly observed in this study, totalling only $\sim 20 \%$ for $>10 \mathrm{keV}$. These ions are observed most frequently in the centre of the range of invariant latitudes observed here, implying that these $L$-shells are the most fertile locations for wave growth to occur.

Conjugate observations, of high $m$ ULF waves in the morning sector, and ion distribution function data revealing a positive gradient region are limited to one case study, presented in Baddeley et al. (2002), which showed an energy location of $\sim 10 \mathrm{keV}$ for the positive gradient region. There is in addition, however, a study where ion data containing a bump-on-tail feature, located at $\sim 7-10 \mathrm{keV}$, was seen $2 \mathrm{~h}$ prior to ionospheric and magnetometer observations of a ULF wave (Wright et al., 2001). In both cases the particle location of the bump-on-tail feature is in the low energy range, in agreement with this study.

Engebretson et al. (1992) investigated a number of radially polarised ULF wave events in the frequency range $5-15 \mathrm{mHz}$ using AMPTE/CCE, GOES 5 and GOES 6. They concluded that 21 observed pulsation events, seen on the day side and primarily in the dusk sector, were Pc4 events driven through interactions with $100 \mathrm{keV}$ protons. More recently Yeoman and Wright (2001) observed high $m$ Pc4 waves in the afternoon sector utilising the CUTLASS radars suggesting the waves were driven by drift and drift-bounce resonance from particle of energy $37-57 \mathrm{keV}$ and $35-41 \mathrm{keV}$ respectively. While the energies given by Yeoman and Wright (2001) are clearly available, through abundant likely ion populations at the lowest invariant latitudes covered by this study in the afternoon sector, only $\sim 2 \%$ of observed IDFs contain $>10^{10} \mathrm{~J}$ at the higher energy locations of $\sim 100 \mathrm{keV}$. Almost all such, rare, events are found between 18:00 and 21:00 MLT at the lowest invariant latitudes. Overall this would suggest that Pc4 events from high energy particles are unlikely to be produced due to positive gradients in the ion distribution functions, but could be related to spatial gradients at these energies.

\section{Summary}

This paper has presented a statistical study of magnetospheric ring current proton populations using 6 years of IDF data gathered using the CAMMICE (MICS) particle instrument. The IDF database created was used to determine the statistically most likely location of positive gradient regions in the ion distributions with respect to MLT, invariant latitude and the proton energy within the IDFs. Furthermore, using the method set out in Baddeley et al. (2004), the free energy contained in such distributions was calculated to ascertain those that could provide enough energy to standing mode structures in the magnetosphere to generate observable high $m$ ULF waves. This study has shown that the most likely candidate unstable, non-Maxwellian populations are low energy protons $(<10 \mathrm{keV})$ located between 10:00 and 19:00 MLT at invariant latitudes $<68^{\circ}$. In contrast protons of energies $\sim 7-40 \mathrm{keV}$ dominate from invariant latitudes of $\sim 66^{\circ}$ to $70^{\circ}$, with a focus at $\sim 7 \mathrm{keV}$, at 05:00-12:00 MLT. The dominant region in the morning sector is consistent with the results of Baddeley et al. (2004), which looked at all data contained between $L$-shell 6 and 9. Above $71^{\circ}$ the number of bump-on-tail features falls rapidly as the number of closed field lines able to support trapped particles that make up the studied IDFs become less commonly available. The invariant latitude range of the ion populations dominant in the morning sector is conjugate to the HF Doppler sounder (DOPE) facility located between $69^{\circ}$ and $70^{\circ}$ geographic latitude. The bump-on-tail populations observed at these invariant latitudes are consistent with the work using DOPE by Yeoman et al. (2000), in finding that the morning sector is a more fertile region for high $m$ ULF wave generation than previously thought. Furthermore, this study would suggest that Pgs are likely to be even mode as there are few candidate ion populations that support enough free energy $\left(>10^{10} \mathrm{~J}\right)$ to allow odd mode generation. In the afternoon sector, around dusk, this study provides evidence to support the work of Allan et al. (1982, 1983); Yeoman and Wright (2001) and Baumjohann et al. (1987) in identifying storm time, compressional Pc5 pulsations as fundamental, odd mode oscillations. These are driven by the ions of $30-60 \mathrm{keV}$ at the lower invariant latitudes studied here.

While this paper has revealed some important relationships in the particle populations of the magnetosphere there 
are some points which could not be addressed. Firstly, the lowest invariant latitudes studied in this paper $\left(\leq 67^{\circ}\right)$ are clearly an extremely abundant area for data, but due to the orbital constraints of Polar and/or the time resolution of CAMMICE (MICS) much of the possible information was removed to maintain the statistical integrity of the study. Having a spacecraft that could remain on the magnetic field lines linked to these invariant latitudes for a longer period of time, or gather larger quantities of data in a short time, would allow a more detailed look at these lower invariant latitudes. This would be of benefit also in that many of the other studies looking at particles or wave event occurrence have been focussed solely below $70^{\circ}$. Secondly, while Polar has provided a wealth of information over its many years of life, it can not tell us many things in relation to the spatial gradient present in the IDFs. For this a multi-spacecraft mission would be more suited such as the Cluster mission as flies currently.

Acknowledgements. The Authors would like to thank T. Fritz, PI of CAMMICE (MICS) and two referees. MEW is supported by a PPARC Quota Studentship.

Topical Editor I. A. Daglis thanks G. Chisham and another referee for their help in evaluating this paper.

\section{References}

Allan, W. and Poulter, E. M.: The spatial structure of different ULF pulsation types: A review of STARE radar results, Rev. Geophys Space Phys., 22, 85-97, 1984.

Allan, W., Poulter, E. M., and Nielsen, E.: STARE observations of a Pc5 pulsation with large azimuthal wave number, J. Geophys. Res., 87, 6163-6172, 1982.

Allan, W., Poulter, E. M., and Nielsen, E.: Pc5 pulsations associated with ring current proton drifts: STARE radar observations, Planet. Space Sci., 31, 1279-1289, 1983.

Anderson, B. J.: Statistical studies of Pc 3-5 pulsations and their relevance for possible source mechanisms of ULF waves, Ann. Geophys., 11, 128-143, 1993,

http://www.ann-geophys.net/11/128/1993/.

Anderson, B. J., Engebretson, M. J., Rounds, S. P., Zanetti, L. J., and Potemra, T. A.: A statistical study of Pc 3-5 pulsations observed by the AMPTE/CCE magnetic fields experiment. I Occurrence distributions, J. Geophys. Res., 95, 10 495-10 523, 1990.

Baddeley, L. J., Yeoman, T. K., Wright, D. M., Davies, J. A., Trattner, K. J., and Roeder, J. L.: Morning sector drift-bounce resonance driven ULF waves observed in artificially induced HF radar backscatter, Ann. Geophys., 20, 1487-1498, 2002, http://www.ann-geophys.net/20/1487/2002/.

Baddeley, L. J., Yeoman, T. K., Wright, D. M., Trattner, K. J., and Kellet, B. J.: A statistical study of unstable particle populations in the global ringcurrent and their relation to the generation of high ULF waves, Ann. Geophys., 22, 4229-4241, 2004, http://www.ann-geophys.net/22/4229/2004/.

Baddeley, L. J., Yeoman, T. K., Wright, D. M., Trattner K. J., and Kellet, B. J.: On the coupling between unstable magnetospheric particle populations and resonant high $m$ ULF wave signatures in the ionosphere, Ann. Geophys., 23, 567-577, 2005, http://www.ann-geophys.net/23/567/2005/.

Baumjohann, W., Sckopke, N., LaBelle, J., and Klecker, B.: Plasma and Field Observations of a Compressional Pc5 Wave Event, J. Geophys. Res., 92, 12 203-12 212, 1987.

Chisham, G.: Giant pulsations: An explanation for their rarity and occurrence during geomagnetically quiet times, J. Geophys. Res., 101, 24 755-24 763, 1996.

Chisham, G. and Orr, D.: Statistical studies of giant pulsations (Pgs): Harmonic mode, Planet. Space Sci., 39, 999-1006, 1991.

Cowley, S. W. H. and Ashour-Abdallah, M.: Adiabatic plasma convection in a dipole field: Proton forbidden-zone effects for a simple electric field model, Planet. Space Sci., 24, 821-833, 1976.

Engebretson, M. J., Murr, D. L., Erickson, K. N., Strangeway, R. J., Klumpar, D. M., Fuselier, S. A., Zanetti, L. J., and Potemra, T. A.: The spatial extent of radial magnetic pulsation events observed in the dayside near synchronous orbit, J. Geophys. Res., 97, 13 741-13 758, 1992.

Hasegawa, A.: Drift mirror instabilities in the magnetosphere, Phys. Fluids, 12, 2642-2650, 1969.

Hughes, W. J., Southwood, D. J., Mauk, B., McPherron, R. L., and Barfield, J. N.: Alfvén waves generated by an inverted plasma energy distribution, Nature, 275, 43-44, 1978.

Lessard, M. R., Hudson, M. K., and Lühr, H.: A statistical study of Pc3-Pc5 magnetic pulsations observed by the AMPTE/Ion Release Module satellite, J. Geophys. Res., 104, 4523-4538, 1999.

Milan, S. E., Yeoman, T. K., Lester, M., Thomas, E. C., and Jones, T. B.: Initial backscatter occurrence statistics from the CUTLASS HF radars, Ann. Geophys., 15, 703-718, 1997, http://www.ann-geophys.net/15/703/1997/.

Nielsen, E., Guttler, W., Thomas, E. C., Stewart, C. P., Jones, T. B., and Hedburg, A.: SABRE - new radar-auroral backscatter experiment, Nature, 304, 712-714, 1983.

Ozeke, L. G. and Mann, I. R.: Modeling the properties of high $m$ Alfvén waves driven by the drift-bounce resonance mechanism, J. Geophys. Res., 106, 15 583-15 597, 2001.

Pokhotelov, O. A., Pilipenko, V. A., Nezlina, Y. M., Woch, J., Kremser, G., North, A., and Amata, E.: Excitation of high $\beta$ plasma instabilities at the geostationary orbit - theory and observations, Planet. Space Sci., 34, 695-712, 1986.

Robinson, T. R., Yeoman, T. K., Dhillon, R. S., Lester, M. E., Thomas, C. J., Thornhill, D., Wright,D. M., van Eyken, A. P., and McCrea, I. W.: First observations of SPEAR induced artificial backscatter from CUTLASS and the EISCAT Svalbard radars, Ann. Geophys., in press, 2006.

Rodriguez, P., Kennedy, E. J., Keskinen, M. J., Siefring, C. L., Basu, S., McCarrick, M., Preston, J., Engebretson, M. J., Kaiser, M. L., Desch, M. D., Goetz, K., Bougeret, J.-L., and Manning, R.: The WIND-HAARP experiment: Initial results of high power radiowave interactions with space plasmas, Geophys. Res. Lett., 25, 257-260, 1998.

Southwood, D. J.: A general approach to low-frequency instability in the ring current plasma, J. Geophys. Res., 81, 3340-3348, 1976.

Southwood, D. J., Dungey, J. W., and Etherington, R. J.: Bounce resonant interactions between pulsations and trapped particles, Planet. Space Sci., 17, 349-361, 1969.

Takahashi, K.: ULF waves: 1997 IAGA division 3 reporter review, Ann. Geophys., 16, 787-803, 1998, 
http://www.ann-geophys.net/16/787/1998/.

Takahashi, K., Fennell, J. F., Amata, E., and Higbie, P. R.: Fieldaligned structure of the storm time Pc5 wave of November 1415, 1979, J. Geophys. Res., 92, 5857-5864, 1987.

Thompson, S. M. and Kivelson, M. G.: New Evidence for the origin of giant pulsations, J. Geophys. Res., 106, 21 237-21 253, 2001.

Tsyganenko, N. A.: A magnetospheric magnetic field model with a warped tail current sheet, Planet. Space Sci., 37, 5-20, 1989.

Wilken, B., Weiß, W., Hall, D., Grande, M., Sørass, F., and Fennell, J. F.: Magnetospheric Ion Composition Spectrometer on board the CRRES spacecraft, J. Spacecraft Rockets, 29, 585591, 1992.

Woch, J., Kremser, G., and Korth, A.: A comprehensive investigation of compressional ULF waves observed in the ring current, J. Geophys. Res., 95, 15 113-15 132, 1990.

Wright, D. M., Yeoman, T. K., and Chapman, P. J.: High-latitude HF Doppler observations of ULF waves. 1. Waves with large spatial scale sizes, Ann. Geophys., 15, 1548-1556, 1997,

http://www.ann-geophys.net/15/1548/1997/.
Wright D. M., Yeoman, T. K., Rae, I. J., Storey, J., Stockton-Chalk, A. B., Roeder, J. L., and Trattner, K. J.: Ground-based and Polar spacecraft observations of a giant $(\mathrm{Pg})$ pulsation and its associated source mechanism, J. Geophys. Res., 106, 10 837-10 852, 2001.

Yeoman, T. K. and Wright, D. M.: ULF waves with drift resonance and drift-bounce resonance energy sources as observed in artificially-induced HF radar backscatter, Ann. Geophys., 19, 159-170, 2001, http://www.ann-geophys.net/19/159/2001/.

Yeoman, T. K., Tian, M., Lester, M., and Jones, T. B.: A study of Pc5 hydromagnetic waves with equatorward phase propagation, Planet. Space Sci., 40, 797-810, 1992.

Yeoman, T. K., Wright, D. M., Chapman, P. J., and Stockton-Chalk, A. B.: High-latitude observations of ULF waves with large azimuthal wavenumbers, J. Geophys. Res., 105, 5453-5462, 2000. 